\title{
Toward Broadband Plasmonics: Tuning Dispersion in Rhombic Plasmonic Crystals
}

\author{
Wei Zhou, ${ }^{\dagger}$ Hanwei Gao, ${ }^{\ddagger}$ and Teri W. Odom ${ }^{+, \neq, *}$ \\ ${ }^{\dagger}$ Department of Materials Science and Engineering, and `Department of Chemistry, Northwestern University, 2145 Sheridan Road, Evanston, Illinois 60208-3113
}

S urface plasmon polaritons (SPPs) are collective charge oscillations coupled to light that propagate along a metaldielectric interface. One strategy to achieve efficient SPP excitation is to pattern the metal surface; then, the momentum mismatch between SPPs and free-space light can be bridged by Bragg vectors inherent in the periodic nanostructures. ${ }^{1,2}$ The intense, localized electromagnetic (EM) fields associated with SPPs can be used to manipulate and enhance light-matter interactions at subwavelength scales. ${ }^{3}$ Hence, periodic metallic nanostructures (so-called plasmonic crystals in two dimensions (2D) ${ }^{4}$ ) are considered as promising plasmonic components for thin-film photovoltaics, ${ }^{5}$ light emitting devices, ${ }^{6,7}$ optical switches, ${ }^{8}$ label-free sensors, ${ }^{9-11}$ and negative index metamaterials. ${ }^{12}$

Recent advances in nanofabrication techniques such as e-beam lithography, focused ion beam (FIB) milling, soft nanoimprint lithography, and soft interference lithography (SIL) have revived interest in plasmonic crystals. ${ }^{13-17}$ Periodic structures with subwavelength feature sizes $(<200$ $\mathrm{nm})$ on small pitches $\left(a_{0}=400-600 \mathrm{~nm}\right)$ can now be obtained and which are necessary to generate low-order Bragg resonances at visible wavelengths. The amplitudes of low-order resonances are stronger than high-order ones because the probability of scattering by single Bragg vectors is much larger than multiple scattering events. ${ }^{18}$ Moreover, SIL can easily pattern arrays of nanostructures in parallel, over wafer-scale areas, and in 2D. Compared to one-dimensional (1D) gratings, twodimensional arrays offer more flexibility to optimize and tune resonances from SPPs propagating in different directions and are www.acsnano.org
ABSTRACT The angle-dependent optical properties of rhombic plasmonic crystals are described. We show that by extending the capabilities of soft interference lithography, subwavelength periodic patterns with arbitrary 2D Bravais lattices can be generated. In addition, we demonstrate that by lowering the plasmonic crystal lattice symmetry, degenerate conditions can be lifted and more plasmon bands can be excited within a fixed wavelength range. Degeneracies were also removed by changing the polar and azimuthal angles of excitation and visualized in dispersion diagrams. Anticrossings between different plasmon bands were observed to depend significantly on the local refractive index and the excitation direction.

KEYWORDS: plasmonic crystal · dispersion diagram - rhombic lattice $\cdot$ anticrossings $\cdot$ band gaps

advantageous for applications that require light-matter interactions over a large solid angle.

Most work on plasmonic crystals has focused on highly symmetric lattices such as square and hexagonal lattices. ${ }^{13,14,19}$ There are, however, three other types of Bravais lattices in 2D: oblique ( $p 2)$, rectangular $(\mathrm{pmm})$, and rhombic $(\mathrm{cmm})$. Because of the fabrication challenges in creating largearea, subwavelength plasmonic crystals with arbitrary lattice symmetries, there are few reports on how the lattice symmetry affects the optical properties. In addition, the two excitation angles $(\theta$, the polar angle relative to normal incidence, and $\phi$, the azimuthal angle relative to a specific lattice direction) affect the SPP-light coupling process, but there have been few systems investigated out to large $\theta$ and $\phi$ because the patterned areas were not large enough. Recent work has demonstrated that changes in $\phi$ can have a significant effect on the properties of $1 \mathrm{D}$ gratings and can be adjusted to optimize the sensing performance; ${ }^{20}$ however, $\phi$-dependent optical properties have not been investigated in 2D plasmonic crystals.

Here we report a variation of SIL that can produce $\mathrm{cm}^{2}$-area rhombic plasmonic

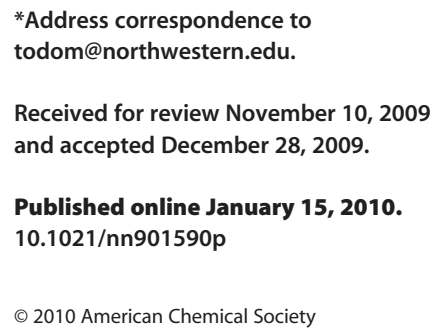
todom@northwestern.edu.

Received for review November 10, 2009 and accepted December 28, 2009.

Published online January 15, 2010. $10.1021 / \mathrm{nn} 901590$ p

() 2010 American Chemical Society 


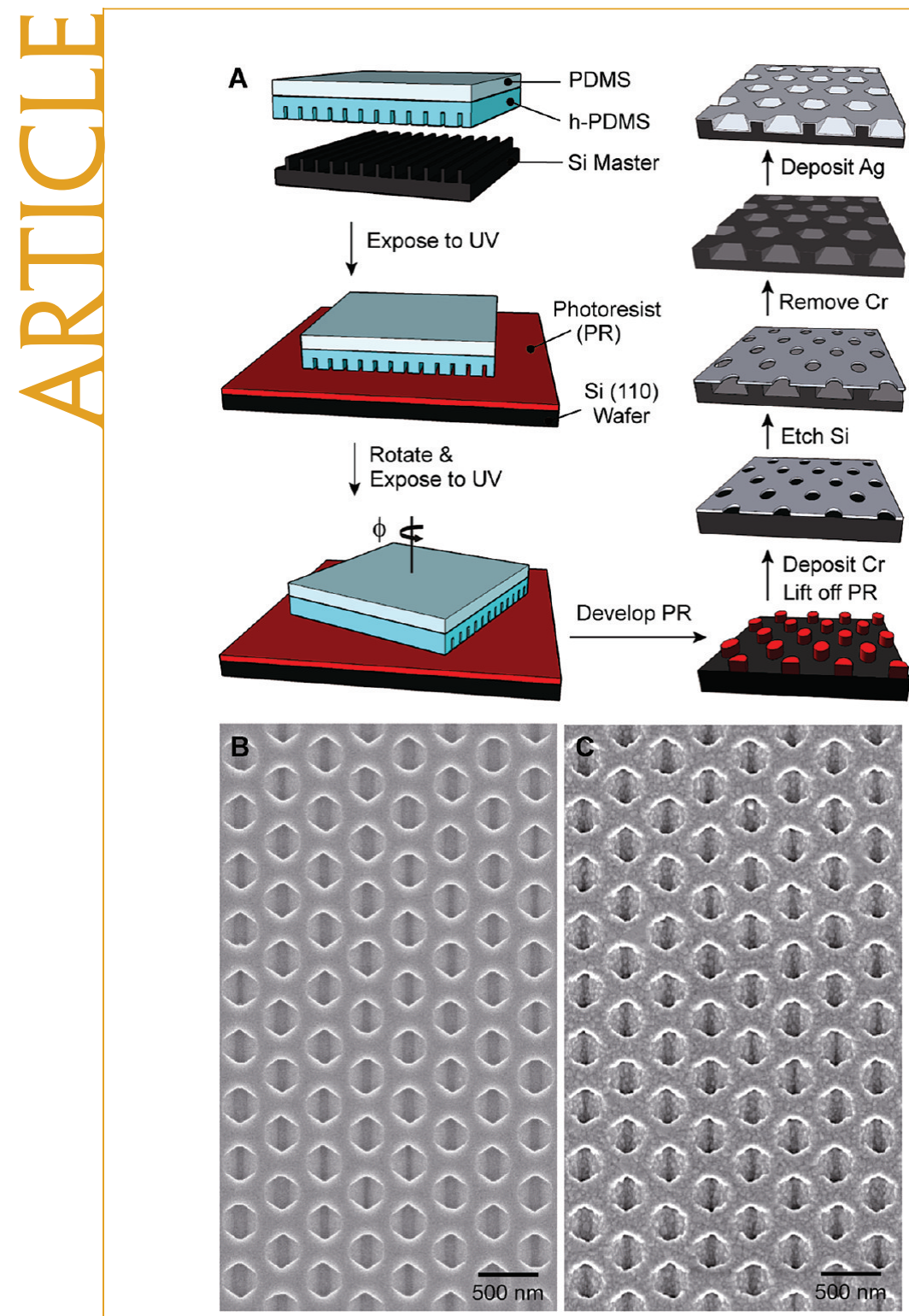

Figure 1. (A) Scheme for fabricating rhombic plasmonic crystals; (B) scanning electron microscopy (SEM) image of a Si template patterned with a rhombic lattice; (C) SEM image of Ag rhombic plasmonic crystals.

crystals. We demonstrate that a large number of plasmon resonances can be generated in the visible range by lowering the lattice symmetry and/or aligning the light excitation along low-symmetry crystal directions. These results offer a new route toward broadband plas-
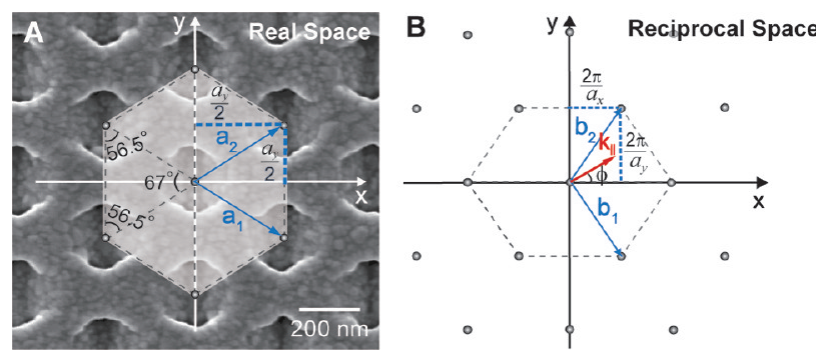

Figure 2. (A) Real space lattice vectors superimposed on a $\mathrm{Ag}$ rhombic plasmonic crystal. $\mathbf{a}_{1}$ and $\mathbf{a}_{2}$ are the primitive vectors with magnitudes $a_{x}=728 \mathrm{~nm}$ and $a_{y}=480 \mathrm{~nm}$. (B) First Brillouin zone with reciprocal lattice vectors $\mathbf{b}_{1}$ and $\mathbf{b}_{2}$.

monics by (i) engineering lattice symmetries to lift SPP mode degeneracies, and (ii) controlling independently the two excitation angles $(\theta, \phi)$. Also, interesting physical phenomena, such as anticrossings between SPP bands, and band gaps at Brillion zone boundaries, were observed to depend strongly on the local refractive index and the excitation direction.

\section{RESULTS AND DISCUSSION}

Figure 1A depicts the fabrication scheme to create rhombic plasmonic crystals. First, a SIL-line photomask was produced by molding PDMS ${ }^{21}$ against a high quality Si master patterned with an array of $1 \mathrm{D}$ ridges (width $d=100 \mathrm{~nm}$, height $h=400 \mathrm{~nm}$, pitch $a_{0}=400 \mathrm{~nm}$ ). Next, the SIL mask was placed in conformal contact with a positive-tone photoresist layer (Shipley 1805, thickness $t=100 \mathrm{~nm}$ ) on a Si (110) wafer, and two sequential UV exposures (with an angle $\phi=67^{\circ}$ ) were performed. A rhombic array of elliptical photoresist posts resulted after development. After deposition of a thin layer of $\mathrm{Cr}$ (10 nm) and lift-off, the $\mathrm{Si}$ (110) substrate was anisotropically etched in $\mathrm{KOH}_{,}{ }^{15}$ which resulted in hexagonal pits consisting of six $\{111\}$ planes below the perforated Cr film (Supporting Information, Figure S1). The $\mathrm{Cr}$ mask was then removed to reveal an etched $\mathrm{Si}$ template with a rhombic lattice (Figure 1B). We deposited $70 \mathrm{~nm}$ of $\mathrm{Ag}$ on the template to create rhombic plasmonic crystals (Figure 1C). Other types of lattice symmetries can be generated using an SIL-line mask by changing the second exposure angle (Supporting Information, Figure S2) or by performing the second exposure through a different line mask with a different pitch.

Figure 2 depicts the rhombic plasmonic crystal in real space and reciprocal space. The two primitive vectors $\mathbf{a}_{\mathbf{1}}$ and $\mathbf{a}_{\mathbf{2}}$ of the rhombic lattice can be expressed in terms of the unit vectors $\hat{\mathbf{x}}$ and $\hat{\mathbf{y}}$ (Figure $2 \mathrm{~A}$ ), where $\mathbf{a}_{\mathbf{1}}$ $=1 / 2\left(a_{x} \hat{\mathbf{x}}-a_{y} \hat{\mathbf{y}}\right), \mathbf{a}_{2}=1 / 2\left(a_{x} \hat{\mathbf{x}}+a_{y} \hat{\mathbf{y}}\right)$. According the SEM image, $a_{x}=728 \mathrm{~nm}$ and $a_{y}=480 \mathrm{~nm}$. Using the orthogonal relation $\mathbf{a}_{i} \cdot \mathbf{b}_{j}=2 \pi \delta_{i j}{ }^{22}$ the corresponding reciprocal lattice vectors $\mathbf{b}_{\mathbf{1}}$ and $\mathbf{b}_{\mathbf{2}}$ (Figure 2B) can be constructed, where $\mathbf{b}_{1}=2 \pi\left(a_{x}{ }^{-1} \hat{\mathbf{x}}-a_{y}{ }^{-1} \hat{\mathbf{y}}\right), \mathbf{b}_{\mathbf{2}}=2 \pi\left(a_{x}{ }^{-1} \hat{\mathbf{x}}\right.$ $\left.+a_{y}{ }^{-1} \hat{\mathbf{y}}\right)$. Hence the Bragg grating vectors can be expressed as $\mathbf{G}_{i j}=i \mathbf{b}_{\mathbf{1}}+j \mathbf{b}_{\mathbf{2}} \equiv(i, j)$, where $i$ and $j$ are integers.

Figure $3 \mathrm{~A}$ defines the excitation conditions for our system. The SPP Bloch modes generated under the Bragg coupling condition can be described by the following dispersion relation ${ }^{1}$

$$
\frac{\omega}{c} \sqrt{\frac{\varepsilon_{d} \varepsilon_{m}}{\varepsilon_{d}+\varepsilon_{m}}}=\left|\mathbf{k}_{\|}+\mathbf{G}_{i j}\right|
$$

where $\varepsilon_{\mathrm{d}}$ and $\varepsilon_{\mathrm{m}}$ are the relative permittivities of the dielectric and the metal, and $\boldsymbol{k}_{\|}$is the in-plane wavevector of incident light whose magnitude $k_{\|}=k_{0} \sin \theta=$ 
$(\omega / c) \sin \theta$. These SPP Bloch wave modes describe how SPP waves propagate along a periodically patterned metal/dielectric interface. If $\phi=0^{\circ}$ is when $\boldsymbol{k}_{\|}$is along the $x$-axis, the dispersion relation for a rhombic plasmonic crystal can be explicitly expressed as

$\frac{\omega}{c} \sqrt{\frac{\varepsilon_{d} \varepsilon_{m}}{\varepsilon_{d}+\varepsilon_{m}}}=$

$\sqrt{\left(k_{||} \cos \phi+i \frac{2 \pi}{a_{x}}+j \frac{2 \pi}{a_{x}}\right)^{2}+\left(k_{\| \mid} \sin \phi-i \frac{2 \pi}{a_{y}}+j \frac{2 \pi}{a_{y}}\right)^{2}}$

Figure 3B-D illustrate how SPP-Bloch wave mode degeneracies can be lifted either after (i) the crystal symmetry is reduced or (ii) the excitation direction is along a low symmetry direction. For a hexagonal lattice, all six lowest order Bragg vectors have the same magnitude (Figure 3B). Correspondingly, the six lowest order SPP modes are degenerate in energy at normal incidence $\left(k_{\|}=0\right)$. When the excitation direction is along $\phi=0^{\circ}$, the mirror symmetry of the hexagonal lattice requires that the SPP wavevectors $\left(\mathbf{k}_{\mathbf{S P P}}\right)$ of the $(0,1)$ and the $(1,0)$ modes have the same magnitude even when $k_{\|} \neq 0$. As a result, their plasmonic bands are degenerate in the dispersion diagram (Figure 3B, right panel); a similar situation exists for the degeneracy between the $(-1,0)$ and $(0,-1)$ modes. When the hexagonal lattice is reduced in symmetry to a rhombic lattice (Figure $3 C$ ), the six lowest order Bragg vectors no longer have the same magnitude. Four vectors still have the same magnitude as the hexagonal case in Figure 3C, but the other two vectors sit on a higher wavevector circle. These degeneracies are easily visualized in the dispersion diagrams, where there are now two branches of SPP modes at normal incidence $\left(k_{\|}=0\right)$ : one branch is at higher energy, and the other has the same energy as the hexagonal case. The $(0,1)$ and $(1,0)$ modes as well as the $(-1,0)$ and $(0,-1)$ modes remain degenerate when $k_{\|} \neq 0$ because $\mathbf{k}_{\|}$is along a mirror symmetry direction $\left(\phi=0^{\circ}\right)$. Noticeably, the band from the $(-1,-1)$ mode crosses with the bands from the $(0,1) /(1,0)$ modes and the $(0,-1) /(-1,0)$ modes. When $\phi$ deviates from a high symmetry direction (e.g., when $\phi=15^{\circ}$ ), the two sets of degenerate modes split into individual ones when $k_{\|} \neq 0$ (Figure 3D), and all degeneracies have been lifted.

Figure 4 shows two sets of reflection spectra measured in air at different incident angles $\theta$, where $\mathbf{k}_{\|}$was aligned along the two mirror symmetry directions $(\phi=$ $0^{\circ}$ and $90^{\circ}$ ). Dips characteristic of the excitation of SPPs were observed in all reflectance spectra. By matching the calculated positions of the SPPs resonances (eq 2) with the measured spectral features, we could assign specific modes to these dips. $\varepsilon_{m}$ values of $\mathrm{Ag}$ were taken from the literature by Johnson and Christy. ${ }^{23}$ Figure $4 \mathrm{~A}$ shows that when $\phi=0^{\circ}$, a relatively broad dip (cen-
A
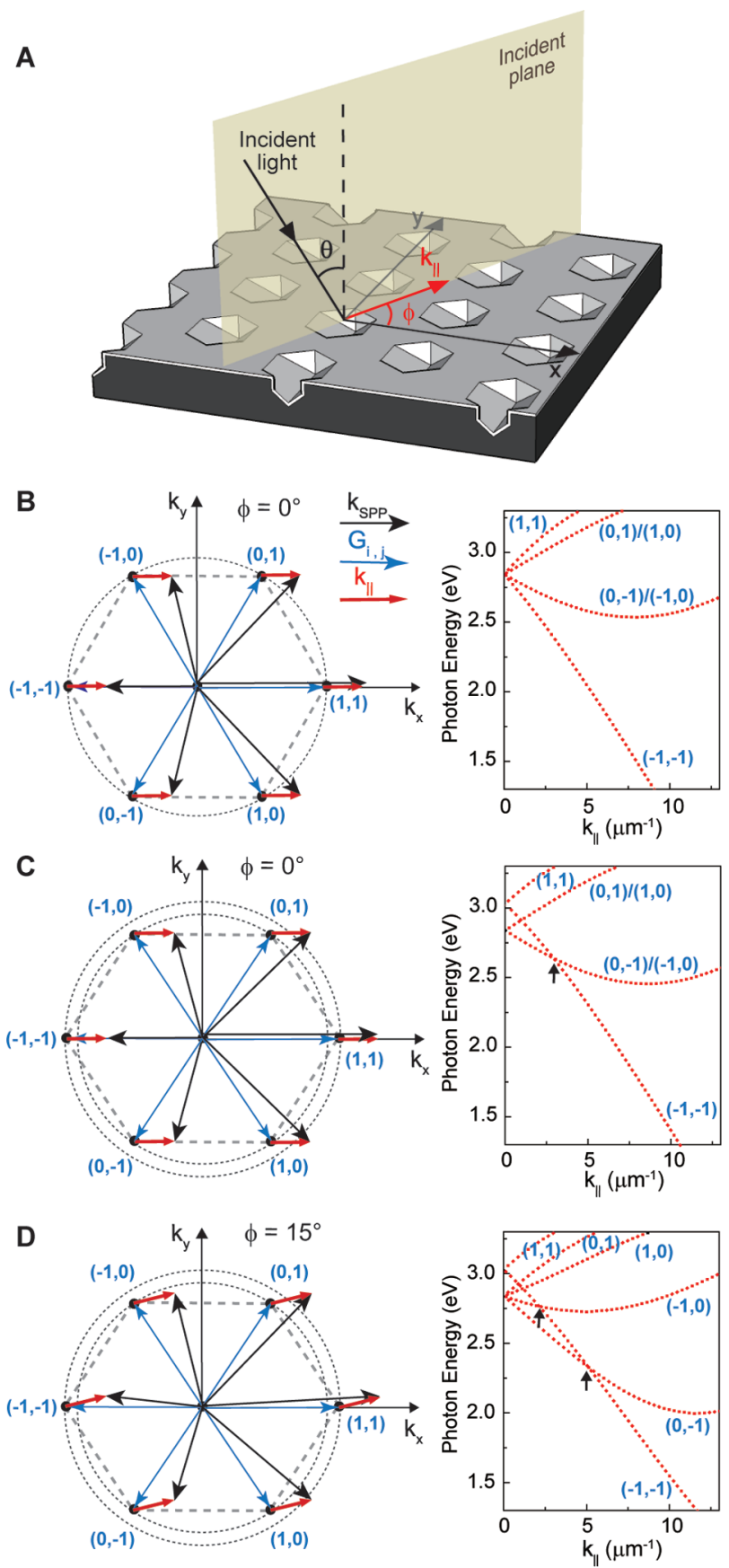

Figure 3. (A) Cartoon defining the orientation direction of incident light $(\theta, \phi)$ with respect to the rhombic lattice. $(B-D)$ Schemes indicating the Bragg excitation of the lowest order SPP modes and their corresponding dispersion curves calculated from eq 2, where (B) is a hexagonal lattice with $\boldsymbol{k}_{\|}$along a high symmetry direction ( $\phi$ $\left.=0^{\circ}\right)$; (C) is a rhombic lattice with $\boldsymbol{k}_{\|}$along a high symmetry direction $\left(\phi=0^{\circ}\right)$; and (D) is a rhombic lattices with $\boldsymbol{k}_{\|}$along a low symmetry direction $\left(\phi=15^{\circ}\right)$. Black arrows indicate the crossing points between different bands.

tered at $460 \mathrm{~nm}$ ) separated into two narrower resonances as $\theta$ increased from $10^{\circ}$ to $60^{\circ}$. The dispersive nature of the $(-1,-1)$ mode, which shifted linearly to longer wavelengths with increasing $\theta$, was very different from the $(-1,0) /(0,-1)$ modes, which shifted from $480 \mathrm{~nm}$ at $\theta=20^{\circ}$ to $502 \mathrm{~nm}$ at $\theta=40^{\circ}$ and then slowly back to $498 \mathrm{~nm}$ at $\theta=60^{\circ}$. Moreover, we found that 

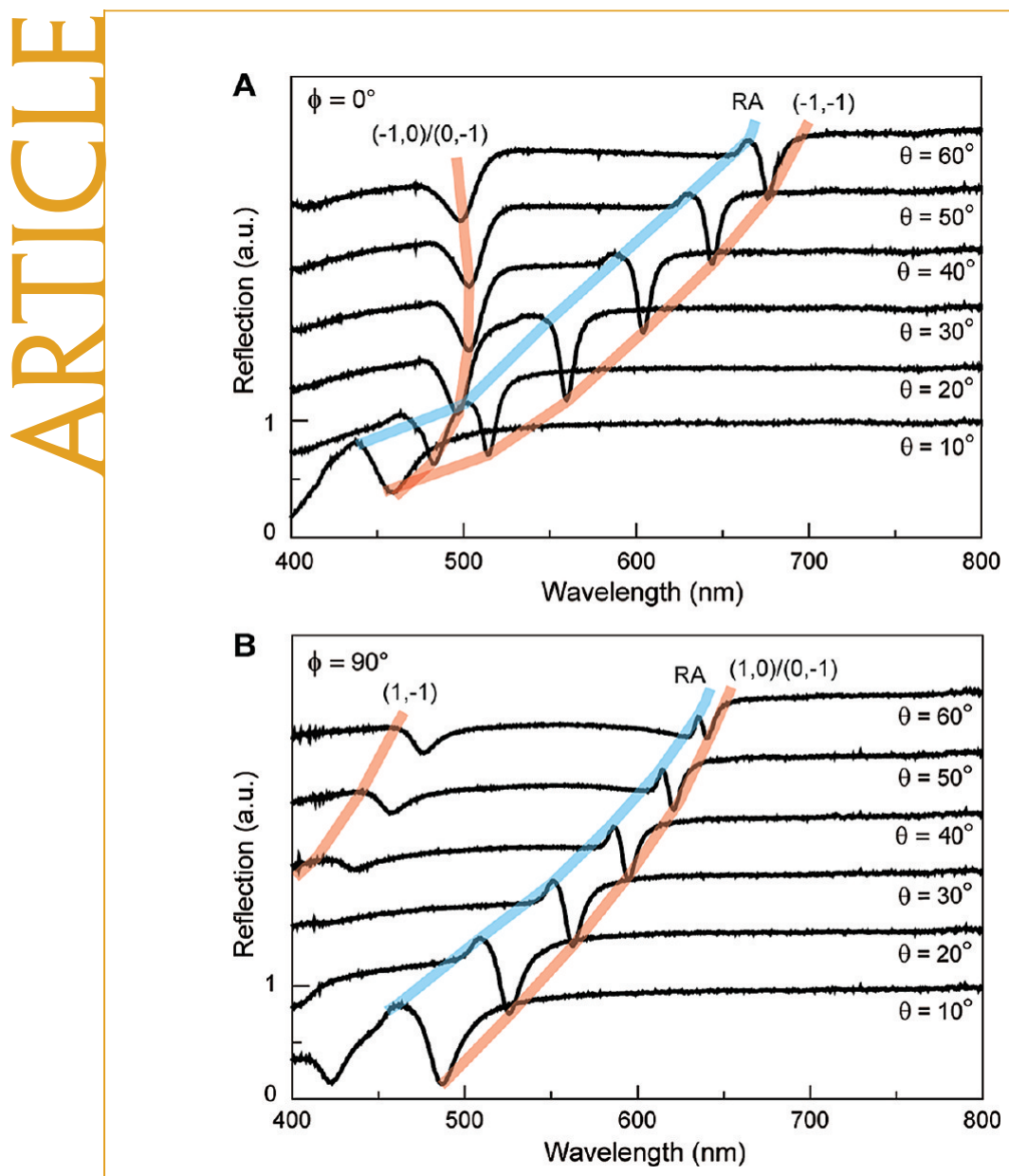

Figure 4. Angle-dependent reflection spectra of Ag rhombic plasmonic crystals with $\mathbf{k}_{\|}$along (A) $\phi=0^{\circ}$ and (B) $\phi=90^{\circ}$. The sample was illuminated in air using $\mathrm{p}$-polarized white light. The red bands represent the position of the SPP modes calculated using eq 2 , and the blue bands denote the position of RAs.

the line width of the $(-1,-1)$ mode was much narrower than that of the $(-1,0) /(0,-1)$ mode at all incident angles. This effect can be attributed to the longer SPP decay time associated with the smaller Rayleighlike scattering cross-section and less electron damping in longer wavelength resonances. ${ }^{24}$ When $\theta$ was greater than $40^{\circ}$, an apparent bump can be observed close to the dip of $(-1,-1)$ mode, which is associated with a Rayleigh anomaly (RA), where the incident light is diffracted parallel to the grating structures (see Supporting Information).

When $\phi$ was changed from $0^{\circ}$ to $90^{\circ}$ (Figure 4B), the reflection spectra showed two dips associated with the $(1,0) /(0,-1)$ and $(-1,0) /(0,1)$ modes at low angles $(\theta$ $\left.=10^{\circ}\right)$. As $\theta$ was increased, the position of the $(1,0) /$ $(0,-1)$ mode monotonically shifted to longer wavelengths, while the position of the $(-1,0) /(0,1)$ mode shifted to shorter wavelengths. The percentage of energy transferred from free-space photons to SPP modes can be estimated by a coupling efficiency $\eta$, which is defined as the depth of the SPP resonance normalized by the reflection intensity baseline. ${ }^{10}$ Although the $(1,0) /$ $(0,-1)$ mode at $\phi=90^{\circ}$ exhibited dispersive trends similar to the $(-1,-1)$ mode at $\phi=0^{\circ}$, its $\eta$ dropped

from a high value $(\sim 85 \%)$ at low angles $\left(\theta=10^{\circ}\right)$ to a much lower one $(\sim 38 \%)$ at $\theta=60^{\circ}$. This observation may be attributed to the SPP propagation direction of the $(1,0) /(0,-1)$ mode shifting away from the polarization direction of the incident light with increasing $\theta$. In contrast, $\eta$ of the $(-1,-1)$ mode at $\phi=0^{\circ}$ changes much slower with increasing $\theta$.

To discriminate among the spectral features in Figure 4, dispersion diagrams were constructed (Figure 5). Each of the measured dispersion diagrams were superimposed with the calculated curves of SPP Bloch wave modes (red, short dashed curves) and RAs (blue, long dashed curves). With $\mathbf{k}_{\|}$along $\mathbf{k}_{\boldsymbol{x}}\left(\phi=0^{\circ}\right)$, two plasmon bands associated with the $(-1,-1)$, and $(-1,0) /(0,-1)$ modes were observed (Figure $5 \mathrm{~A}-\mathrm{C}$ ). These measured bands matched well with the calculated dispersion curves of the SPP modes, especially at high wavevectors. The plasmon band associated with the $(-1,-1)$ mode was very dispersive, while the band associated with the $(-1,0) /(0,-1)$ modes was much less so. The calculated dispersion curves of the $(-1,-1)$ and $(-1,0)$ / $(0,-1)$ Bloch modes crossed at $2.93 \mu \mathrm{m}^{-1}(2.64 \mathrm{eV})$; however, we did not observe a simple overlap of bands in this region (Figure $5 A$ ). Instead, the $(-1,-1)$ band was slightly shifted to lower $E$ compared to the calculated curve, and both bands from the $(-1,-1)$ and $(-1,0) /(0,-1)$ modes were broadened. This observation can be attributed to the anticrossing effect between two coupled SPP modes when they have the same energy and wavevector. ${ }^{25,26}$ Strong coupling between different SPP modes can significantly slow down SPP propagation and increase the SPP density of states. ${ }^{26,27}$ Also, in these anticrossing regions, the nearfield intensity of SPPs can be further enhanced, which is advantageous for sensing applications. Anticrossing phenomena have also been observed in other plasmonic systems between localized surface plasmon (LSP) and SPP modes, ${ }^{24,28}$ SPP and photonic modes, ${ }^{29}$ and photonic and LSP modes. ${ }^{30}$ Although individual pits with sharp edges and corners are likely to support LSPs, we did not observe signature LSP features in the dispersion diagrams. This result suggests that the pits embedded in the continuous metallic-dielectric interface did not act as isolated plasmonic structures. We cannot exclude the possibility, however, that LSP modes were excited initially and then quickly decayed through nearfield coupling and transformed into SPP modes.

By increasing the $n$ of the superstrate, we could resolve two higher energy plasmonic bands: the $(1,1)$ and the $(1,0) /(0,1)$ modes (Figure $5 B-C$ ). Since the propagation direction of the $(1,1)$ mode is along the polarization direction of the incident light (projected on the surface) (Figure 3C), the coupling efficiency $\eta$ of the $(1,1)$ band is much higher than that of the $(1,0) /(0,1)$ band $\left(\eta_{(1,1)} \approx 64 \%\right.$ and $\eta_{(1,0) /(0,1)} \approx 31 \%$ at $\theta=10^{\circ}$ and $n=1.52$ ). Also, in Figure $5 \mathrm{C}$, a clear band gap was observed around $2.54 \mathrm{eV}$ when the SPP wavevector of the 

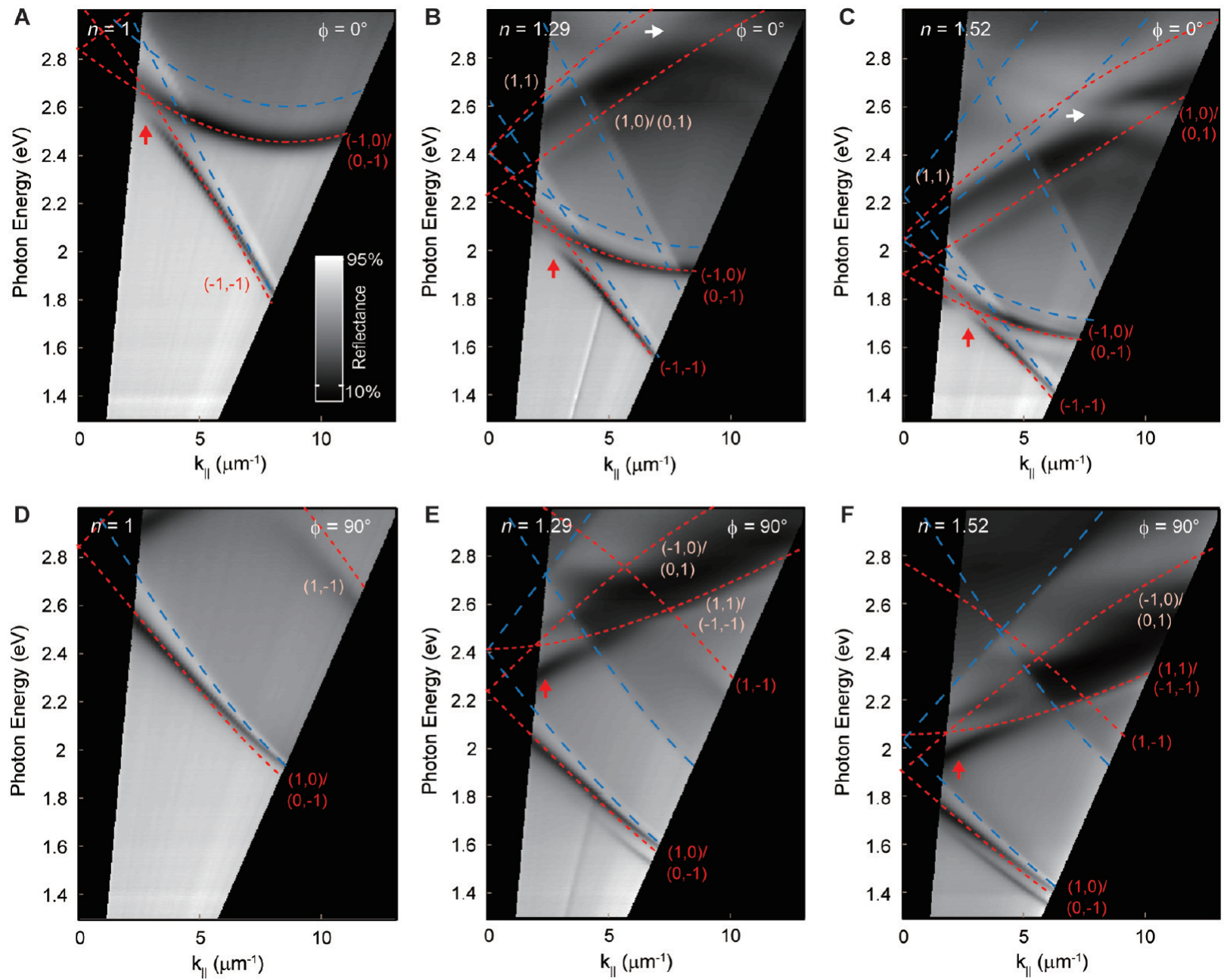

Figure 5. Dispersion diagrams of Ag rhombic plasmonic crystals derived from angle-dependent reflection spectra along high symmetry excitation conditions. (A-C) $\phi=0^{\circ}$, with $n=1.0,1.29,1.52$, and $(D-F) \phi=90^{\circ}$ with $n=1.0,1.29,1.52$. Red, short dashed curves are calculated SPP waves, and blue, long dashed curves are RAs. Red arrows mark the anticrossing regions, and white arrows indicate band gaps at Brillouin zone boundaries.

$(1,1)$ mode crossed a high order Brillouin zone boundary $\left(\left|\mathbf{k}_{\mathrm{SPP}}\right|=\left|\mathbf{G}_{1,1}+\mathbf{k}_{\|}\right|=\left(2 \times 2 \pi / a_{x}\right)+2 \pi / a_{x}=25.8\right.$ $\mu \mathrm{m}^{-1}$ ), where $\mathbf{k}_{\|}=2 \pi / a_{x}=8.6 \mu \mathrm{m}^{-1}$. This band gap is induced by standing waves formed by the interference between forward and backward scattered SPP waves. ${ }^{19}$

Figure 5D shows that when $\mathbf{k}_{\|}$is along $\mathbf{k}_{\mathbf{y}}\left(\phi=90^{\circ}\right)$, the measured dispersion diagram showed bands with shapes different from those at $\phi=0^{\circ}$. By increasing the superstrate $n$, more features related to SPPs and RAs were resolved, and three plasmonic bands associated with the $(1,0) /(0,-1),(1,-1)$, and $(-1,0) /(0,1)$ modes were observed. Anticrossing between the $(-1,0) /(0,1)$ and $(1,1) /(-1,-1)$ modes were observed when the calculated dispersion curves crossed $1.88 \mu \mathrm{m}^{-1}(2.42 \mathrm{eV})$ for $n=1.29$ (Figure 5E) and $1.96 \mu \mathrm{m}^{-1}(2.08 \mathrm{eV}$ ) for $n=$ 1.52 (Figure 5F).

Figure 6 shows two series of dispersion diagrams when $\mathbf{k}_{\|}$is along two low symmetry directions of the rhombic crystal $\left(\phi=35^{\circ}\right.$ and $\left.\phi=55^{\circ}\right)$. As expected, the dispersion properties of the rhombic plasmonic crystals were very sensitive to $\phi$. Previously degenerate www.acsnano.org plasmonic bands $((-1,0) /(0,-1)$ and $(1,0) /(0,1)$ modes for $\phi=0^{\circ} ;(-1,0) /(0,1),(1,0) /(0,-1)$, and $(1,1) /(-1,-1)$ modes for $\phi=90^{\circ}$ ) were no longer so, and more plasmonic bands were observed in the dispersion diagrams. Hence, the amount of energy trapped from the incident light as SPPs in plasmonic crystals not only depends on the structural properties of the lattice but also on the in-plane excitation direction.

Interestingly, we found that the appearance of the anticrossings in the dispersion diagrams depended on the excitation direction of incident light and the superstrate $n$. Figure 6 panels $A-C$ show that when $\phi=35^{\circ}$, the wavevector location of the anticrossing was fixed $\left(k_{\|}=2 \mu \mathrm{m}^{-1}\right)$ but the $E$ changed as a function of the superstrate $n$. Also, the anticrossing between the $(-1,-1)$ and $(-1,0) /(1,0)$ modes produced two bands of similar strength (Figure $6 \mathrm{C}$ ). In contrast, when $\phi=55^{\circ}$, the calculated dispersion curves of both $(-1,-1)$ and $(1,0)$ modes became less dispersive (Figure 6D), and the anticrossing between these two modes produced a stronger band at higher $E$ and lower $k_{\|}$, and a weaker 

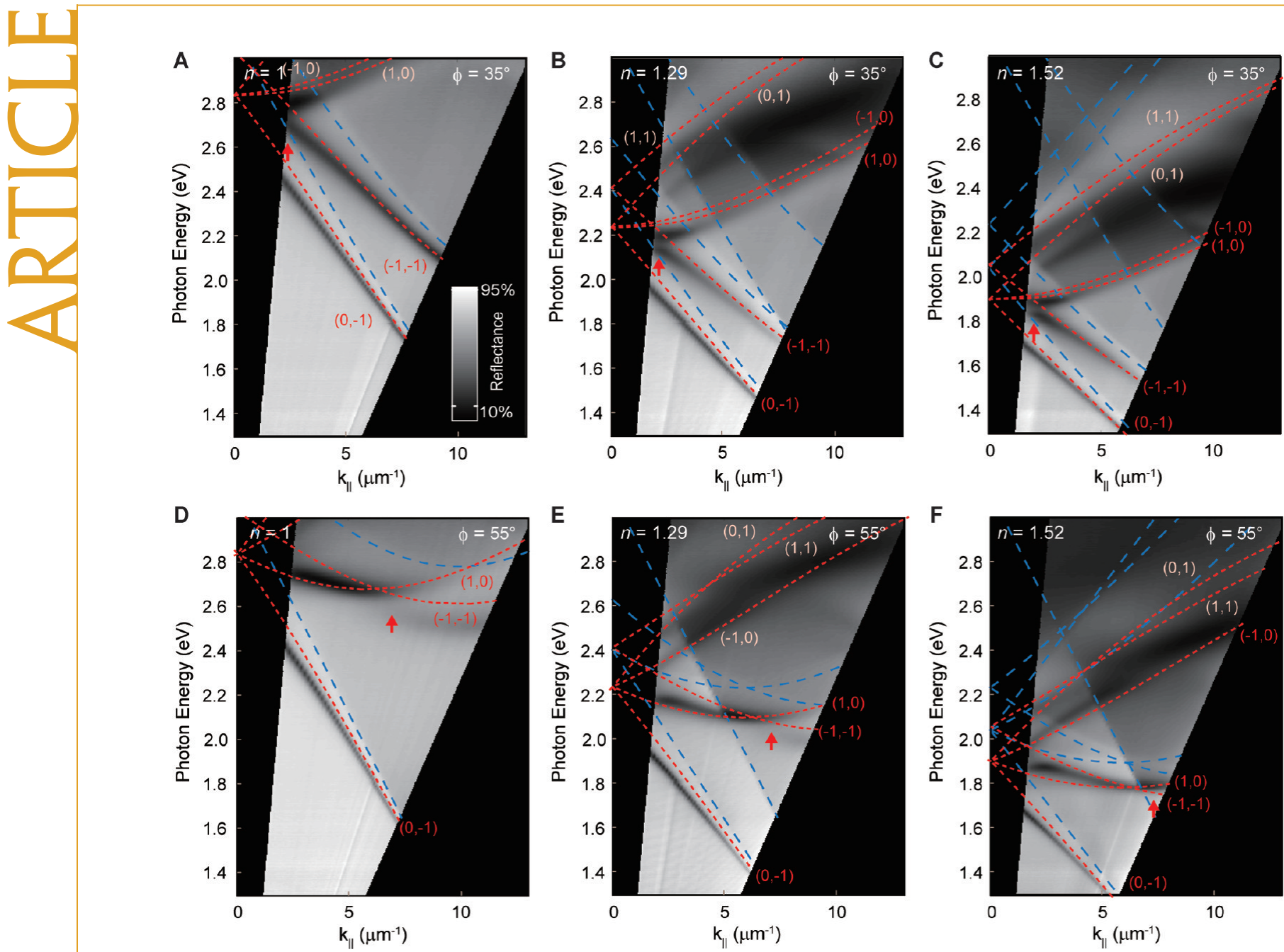

Figure 6. Dispersion diagrams of Ag rhombic plasmonic crystals derived from angle-dependent reflection spectra along low symmetry excitation conditions. (A-C) $\phi=35^{\circ}$, with $n=1.0,1.29,1.52$ and $(D-F) \phi=65^{\circ}$ and same superstrate $n$. Red, short dashed curves are calculated SPP waves, and blue, long dashed curves are RAs. Red arrows mark the anticrossing regions, and white arrows indicate band gaps at Brillouin zone boundaries.

band at lower $E$ and higher $k_{\|}$. Unlike the situation with $\phi=35^{\circ}$, the location of the anticrossing between the $(-1,-1)$ and $(1,0)$ modes shifted significantly to higher values of $k_{\|}$with increasing superstrate $n$ (Figure 6D-F).

\section{CONCLUSIONS}

In summary, we have demonstrated that SIL can be used to create low symmetry plasmonic lattices over $\mathrm{cm}^{2}$-areas. Rhombic plasmonic crystals generated more plasmon modes compared to more symmetric lattices within a fixed wavelength range. Excitation directions along low-symmetry lattice directions also were able to lift degeneracies in the dispersion diagrams to form more resonances. Moreover, anticrossings resulting from coupling between different plasmon modes were observed in the dispersion diagrams; these correspond to regions of slower SPP propagation and further concentration of the localized fields. Because the shapes and positions of these anticrossings are very sensitive to changes in refractive index at optimized excitation directions, we anticipate that they could be useful for chemical and biological sensing. Most importantly, our versatile fabrication method provides new opportunities to design plasmonic crystals with arbitrary 2D lattices so that plasmons can be easily tuned over a broader spectrum.

\section{EXPERIMENTAL SECTION}

Angle-Dependent Optical Measurements. Using a home-built, automated rotational stage, zero-order reflection spectra were measured from $\theta=10^{\circ}$ to $60^{\circ}$ in increments of $1^{\circ}$ for a fixed azimuthal angle $\phi$. Collimated TM-polarized (p-polarized) white light from a halogen lamp (100 W) was used to illuminate the sample. The size of the illumination spot on the sample was 2 $\mathrm{mm}$. The reflected light was collected and coupled into a bundled optical fiber connected to a Czerny-Turner spectrometer (Triax 552/LN $\mathrm{L}_{2}$-Cooled CCD, Horiba Jobin Yvon, Inc.). We used a linear interpolation algorithm in MatLab to convert the measured optical data (wavelength $\lambda$ vs polar angle $\theta$ ) into dispersion diagrams (photon energy $E$ vs in-plane wavevector of light $k_{\|}$). To compare the dispersion properties of rhombic 
plasmonic crystals with $\mathbf{k}_{\|}$along different directions, four different $\phi\left(0^{\circ}, 90^{\circ}, 35^{\circ}\right.$, and $\left.55^{\circ}\right)$ were selected. The angles $\phi=0^{\circ}$ and $90^{\circ}$ correspond to $\mathbf{k}_{\|}$along the two mirror symmetry directions of the rhombic lattice, while $\phi=35^{\circ}$ and $55^{\circ}$ correspond to $\mathbf{k}_{\|}$ along two low symmetry directions. These latter directions were selected because they could be accurately aligned along the edges of the Si (110) substrate. To visualize resonances at longer wavelengths, we measured angle-dependent reflection spectra under different refractive index superstrates ( $n=1$ for air, and $n$ $=1.29$ or $n=1.52$ for oils).

Acknowledgment. This work was supported by the National Science Foundation (NSF) under Grants DMR-0705741 and CMMI-0826219 and by the NSF-MRSEC program at the Materials Research Center under Grant DMR-0520513. This work made use of the NUANCE Center facilities, which are supported by NSFMRSEC, NSF-NSEC, and the Keck Foundation.

Supporting Information Available: Unit cell of rhombic lattice; SEM images of photoresist posts after changing the angle between two exposures of the SIL line mask; Raleigh anomalies on a rhombic lattice. This material is available free of charge via the Internet at http://pubs.acs.org.

\section{REFERENCES AND NOTES}

1. Zayats, A. V.; Smolyaninov, I. I.; Maradudin, A. A. Nanooptics of Surface Plasmon Polaritons. Phys.Rep., Rev. Sect. Phys. Lett. 2005, 408, 131-314.

2. Raether, H. Surface Plasmons; Springer: Berlin, 1988.

3. Barnes, W. L.; Dereux, A.; Ebbesen, T. W. Surface Plasmon Subwavelength Optics. Nature 2003, 424, 824-830.

4. Baudrion, A.-L.; Weeber, J.-C.; Dereux, A.; Lecamp, G.; Lalanne, P.; Bozhevolnyi, S. I. Influence of the Filling Factor on the Spectral Properties of Plasmonic Crystals. Phys. Rev. B 2006, 74, 125406.

5. Ferry, V. E.; Sweatlock, L. A.; Pacifici, D.; Atwater, H. A. Plasmonic Nanostructure Design for Efficient Light Coupling into Solar Cells. Nano Lett 2008, 8, 4391-4397.

6. Wedge, S.; Barnes, W. L. Surface Plasmon-Polariton Mediated Light Emission through Thin Metal Films. Opt Express 2004, 12, 3673-3685.

7. Vuckovic, J.; Loncar, M.; Scherer, A. Surface Plasmon Enhanced Light-Emitting Diode. IEEE J. Quantum Electron. 2000, 36, 1131-1144.

8. Dintinger, J.; Klein, S.; Ebbesen, T. W. Molecule-Surface Plasmon Interactions in Hole Arrays: Enhanced Absorption, Refractive Index Changes, and All-Optical Switching. Adv. Mater. 2006, 18, 1267-1270.

9. Gordon, R.; Sinton, D.; Kavanagh, K. L.; Brolo, A. G. A New Generation of Sensors Based on Extraordinary Optical Transmission. Acc. Chem. Res. 2008, 41, 1049-1057.

10. Gao, H. W.; Henzie, J.; Lee, M. H.; Odom, T. W. Screening Plasmonic Materials Using Pyramidal Gratings. Proc. Natl. Acad. Sci. U.S.A. 2008, 105, 20146-20151.

11. Stewart, M. E.; Mack, N. H.; Malyarchuk, V.; Soares, J. A. N. T.; Lee, T.-W.; Gray, S. K.; Nuzzo, R. G.; Rogers, J. A. Quantitative Multispectral Biosensing and 1D Imaging Using Quasi-3D Plasmonic Crystals. Proc. Natl. Acad. Sci. U.S.A. 2006, 103, 17143-17148.

12. Zhang, S.; Fan, W. J.; Panoiu, N. C.; Malloy, K. J.; Osgood, R. M.; Brueck, S. R. J. Experimental Demonstration of nearInfrared Negative-Index Metamaterials. Phys. Rev. Lett. 2005, 95, 137404.

13. Ebbesen, T. W.; Lezec, H. J.; Ghaemi, H. F.; Thio, T.; Wolff, P. A. Extraordinary Optical Transmission through SubWavelength Hole Arrays. Nature 1998, 391, 667-669.

14. Bozhevolnyi, S. I.; Erland, J.; Leosson, K.; Skovgaard, P. M. W.; Hvam, J. M. Waveguiding in Surface Plasmon Polariton Band Gap Structures. Phys. Rev. Lett. 2001, 86, 3008-3011.

15. Gao, H. W.; Henzie, J.; Odom, T. W. Direct Evidence for Surface Plasmon-Mediated Enhanced Light Transmission through Metallic Nanohole Arrays. Nano Lett. 2006, 6, 2104-2108.
16. Henzie, J.; Lee, M. H.; Odom, T. W. Multiscale Patterning of Plasmonic Metamaterials. Nat. Nanotechnol. 2007, 2, 549-554.

17. Malyarchuk, V.; Hua, F.; Mack, N. H.; Velasquez, V. T.; White, J. O.; Nuzzo, R. G.; Rogers, J. A. High Performance Plasmonic Crystal Sensor Formed by Soft Nanoimprint Lithography. Opt Express 2005, 13, 5669-5675.

18. Watts, R. A.; Harris, J. B.; Hibbins, A. P.; Preist, T. W.; Samples, J. R. Optical Excitation of Surface Plasmon Polaritons on 90 Degrees and 60 Degrees Bi-gratings. J. Mod. Optic. 1996, 43, 1351-1360.

19. Kitson, S. C.; Barnes, W. L.; Sambles, J. R. Full Photonic Band Gap for Surface Modes in the Visible. Phys. Rev. Lett. 1996, 77, 2670-2673.

20. Romanato, F.; Hong, L. K.; Kang, H. K.; Wong, C. C.; Yun, Z.; Knoll, W. Azimuthal Dispersion and Energy Mode Condensation of Grating-Coupled Surface Plasmon Polaritons. Phys. Rev. B 2008, 77, 245435.

21. Odom, T. W.; Thalladi, V. R.; Love, J. C.; Whitesides, G. M. Generation of 30-50 nm Structures Using Easily Fabricated, Composite PDMS Masks. J. Am. Chem. Soc 2002, 124, 12112-12113.

22. Kittel, C. Introduction to Solid State Physics; Wiley: New York, 2004

23. Johnson, P. B.; Christy, R. W. Optical-Constants of NobleMetals. Phys. Rev. B 1972, 6, 4370-4379.

24. Chu, Y. Z.; Crozier, K. B. Experimental Study of the Interaction between Localized and Propagating Surface Plasmons. Opt. Lett. 2009, 34, 244-246.

25. Alleyne, C. J.; Kirk, A. G.; McPhedran, R. C.; Nicorovici, N. A. P.; Maystre, D. Enhanced SPR Sensitivity Using Periodic Metallic Structures. Opt. Express. 2007, 15, 81638169.

26. Billaudeau, C.; Collin, S.; Sauvan, C.; Bardou, N.; Pardo, F.; Pelouard, J. L. Angle-Resolved Transmission Measurements through Anisotropic Two-Dimensional Plasmonic Crystals. Opt. Lett. 2008, 33, 165-167.

27. Paiella, R. Tunable Surface Plasmons in Coupled MetalloDielectric Multiple Layers for Light-Emission Efficiency Enhancement. Appl. Phys. Lett. 2005, 87, 111104.

28. Ghoshal, A.; Divliansky, I.; Kik, P. G. Experimental Observation of Mode-Selective Anticrossing in SurfacePlasmon-Coupled Metal Nanoparticle Arrays. Appl. Phys. Lett. 2009, 94, 171108.

29. Ditlbacher, H.; Galler, N.; Koller, D. M.; Hohenau, A.; Leitner, A.; Aussenegg, F. R.; Krenn, J. R. Coupling Dielectric Waveguide Modes to Surface Plasmon Polaritons. Opt. Express 2008, 16, 10455-10464.

30. Christ, A.; Tikhodeev, S. G.; Gippius, N. A.; Kuhl, J.; Giessen, $\mathrm{H}$. Waveguide-Plasmon Polaritons: Strong Coupling of Photonic and Electronic Resonances in a Metallic Photonic Crystal Slab. Phys. Rev. Lett. 2003, 91, 183901. 